\title{
Entre contre-discours et légitimation : la construction d'un ethos testimonial dans L'étrange défaite de Marc Bloch
}

Between Counter-Discourse and Legitimization: the Construction of a

Testimonial Ethos in Strange Defeat by Marc Bloch

\section{Jonathan Sitbon}

\section{(2) OpenEdition \\ Journals}

Édition électronique

URL : http://journals.openedition.org/aad/2593

DOI : 10.4000/aad.2593

ISSN : 1565-8961

Éditeur

Université de Tel-Aviv

Référence électronique

Jonathan Sitbon, «Entre contre-discours et légitimation : la construction d'un ethos testimonial dans L'étrange défaite de Marc Bloch », Argumentation et Analyse du Discours [En ligne], 21 | 2018, mis en ligne le 15 octobre 2018, consulté le 23 septembre 2019. URL : http://journals.openedition.org/aad/ 2593 ; DOI : 10.4000/aad.2593

Ce document a été généré automatiquement le 23 septembre 2019.

\section{(c)}

Argumentation \& analyse du discours est mis à disposition selon les termes de la licence Creative Commons Attribution - Pas d'Utilisation Commerciale - Pas de Modification 4.0 International. 


\title{
Entre contre-discours et
}

\section{légitimation : la construction d'un ethos testimonial dans L'étrange défaite de Marc Bloch}

\author{
Between Counter-Discourse and Legitimization: the Construction of a \\ Testimonial Ethos in Strange Defeat by Marc Bloch
}

Jonathan Sitbon

\section{Introduction}

1 C'est entre juillet et septembre 1940, après la débâcle des armées françaises du Nord, et alors qu'il est réfugié en zone libre avec sa famille, que Marc Bloch rédige L'étrange défaite. L'ouvrage ne sera publié qu'en 1946 par les éditions Franc-Tireur, deux ans après l'assassinat de l'historien par la Gestapo. Entre sa rédaction et sa publication, le manuscrit connaît de nombreuses péripéties, comme le rappelle le géographe Philippe Arbos, auquel il avait été confié (cité dans Bloch 1990 : 9). Le texte est d'abord caché dans la propriété du docteur Pierre Canque, à Clermont-Ferrand. Il est découvert par une patrouille allemande ayant réquisitionné la demeure, mais son contenu n'attire pas leur attention. Canque peut ainsi le récupérer et l'enfouir dans son jardin. À la Libération, il est finalement déterré puis rendu à la famille de Marc Bloch, qui entreprend de le faire connaître au grand public.

2 Cet ouvrage décrit la défaite éclair de l'armée française, en analyse de façon extrêmement minutieuse les causes et en attribue les responsabilités - de l'État-major aux populations de "l'arrière », en passant par la bourgeoisie, les syndicats ouvriers ou encore l'enseignement universitaire de l'époque. L'étrange défaite constitue ainsi un contre-discours, dont l'un des objectifs est de rétablir la vérité face à la propagande officielle alors en vigueur. Pour l'auteur, ce travail s'inscrit en outre dans le genre du 
"témoignage ", et c'est d'ailleurs ainsi qu'il était originellement destiné à s'appeler avant que la maison d'édition, en raison de la publication d'un autre livre portant le même nom, n'en change le titre.

3 «Récit certifié par la présence à l'événement raconté» (Dulong 1998: 11), un témoignage est, par essence, inséparable de l'individu qui le rapporte. Conséquence de cette association : si la valeur du témoin est mise en doute, que ce soit pour des raisons morales (mensonge, mauvaise foi, prise d'intérêt, etc.) ou cognitives (mémoire incertaine, défauts d'élocution ou de narration, etc.), c'est la déposition elle-même qui peut être discréditée. C'est pourquoi « un témoin, pour en gagner le titre, doit offrir des garanties en termes de véracité et de légitimité » (Fleury et Walter 2012:158). Ce sont ces garanties que va tâcher d'apporter Marc Bloch en élaborant, dans les sept premiers paragraphes de la première partie de l'ouvrage (qui constituent notre corpus), un ethos discursif de témoin valable.

4 Pour ce faire, l'historien va déployer une argumentation satisfaisant à une conception traditionnelle du témoignage, issue du droit et de la rhétorique. Parce qu'un témoignage est une preuve (Danblon 2010 : 219), c'est-à-dire un instrument visant à établir une vérité, Bloch va démontrer sa capacité à discerner et à dire le vrai, et ce, malgré les conséquences funestes qui pourraient en résulter pour sa personne. Au cours de son argumentation, il va également être amené à se situer par rapport à son identité juive, qu'il sait connue de ses allocutaires potentiels. Cette intrusion du fait religieux dans un discours sur la défaite militaire française a de quoi étonner. Pourquoi un témoin juif serait-il, dans ce cadre précis, différent d'un autre témoin? Quels moyens argumentatifs doit-il mettre en œuvre pour préserver son droit de parler?

Entre contre-discours et légitimation, le présent article mobilise les outils forgés par l'analyse du discours pour répondre à ces questions et, par là-même, jeter un éclairage nouveau sur les mécanismes de revendication, de construction et de limitation d'un ethos discursif de témoin face à des stéréotypes.

\section{Le témoignage de L'étrange défaite : un contre-discours à légitimer}

\section{Le témoignage comme contre-discours}

6 L'ouvrage de Marc Bloch est truffé de références au genre dans lequel il prétend s'inscrire. Comme nous l'avons déjà évoqué, le titre lui-même devait originellement être Témoignage. Toujours dans le péritexte, la table des matières se décompose en trois parties, la première incorporant explicitement le mot « témoin » et les deux autres des termes apparentés (« déposition » et « examen de conscience »), appartenant eux aussi au champ sémantique du droit, duquel est directement issu le témoignage (Mausen et Gomart 2000 : 69).

7 Le témoignage apparaît de nouveau dès les premières lignes du texte. Dans le chapitre intitulé "Présentation du témoin », Bloch élabore en effet un véritable métadiscours, qui le conduit à utiliser à trois reprises le terme de "témoignage ", au singulier et au pluriel, pour qualifier son entreprise, et celui de «témoin » pour désigner sa propre personne. Le vocabulaire judiciaire est également sollicité pour enrichir la description du genre discursif, avec l'emploi de "procès-verbal », d'« exact bilan » ou d'« état- 
civil ». Plutôt que de le concurrencer, ces termes viennent renforcer l'emploi du qualificatif principal, dans la mesure où ils évoquent des qualités similaires aux siennes : précision, exhaustivité, souci de la vérité, caractère procédural.

Ce travail positif de qualification s'accompagne d'une entreprise d'évacuation des genres auxquels le discours n'appartient pas. Ainsi, Bloch précise qu'il «n'écrit pas [ses] souvenirs ", les " petites aventures personnelles d'un soldat» important peu. De façon péjorative, il rejette également la recherche du « chatouillement du pittoresque ou de l'humour " en cette période qui n'est pas avare de "soucis », prenant ainsi ses distances avec une littérature du témoignage qui fut abondante après la Première Guerre mondiale, et dont les auteurs acceptent d'autant plus facilement le " pathétique des petits récits » (Henri Barbusse, cité et analysé par R. Amossy, 2016 : 102-105) qu'ils se vivent comme les porte-paroles d'une génération.

Inversement, Marc Bloch se montre beaucoup moins regardant sur la notion d'« étude ", qu'il utilise sans plus de justification, alors même qu'elle pourrait être interprétée comme concurrente de celle de témoignage, dans le cadre du travail de définition du genre de son discours. Cette labilité sémantique n'est possible que parce que, si Bloch prend soin de qualifier son texte de témoignage, son métadiscours ne formalise pas les raisons de ce choix, ni ne définit de façon rigoureuse ce qu'est un témoignage. Tout au plus présente-t-il, sans les démontrer, quatre caractéristiques du témoignage qui, aussi peu nombreuses qu'elles soient, n'en sont pas moins essentielles pour l'argumentation.

Première caractéristique: le témoignage n'est valable que s'il est "fixé dans sa première fraîcheur ». En effet, témoigner exige de solliciter sa mémoire, et cette dernière est d'autant moins fiable que les événements relatés sont éloignés dans le temps. Il faut donc parler ou écrire rapidement, afin de garantir autant que possible l'exactitude des faits rapportés. Naturellement, et c'est la deuxième caractéristique du témoignage, ce travail de remémoration et de narration demande un "effort ». Celui auquel Bloch se livre ici est qualifié de « rude », un adjectif fort, qui étonne venant d'un auteur qui a déjà publié de copieux ouvrages scientifiques. Ce n'est donc pas tant le travail d'écriture lui-même qui est désigné, mais les conditions dans lesquelles il a été réalisé. Dans les toutes premières lignes, l'auteur suggère ainsi, sans le dépeindre explicitement, le caractère dramatique de la vie qu'il mène au moment de la rédaction, entre danger, épuisement physique et nerveux, et dissimulation. Et si l'effort est consenti, c'est parce qu'il est nécessaire : la suggestion des conditions de production du texte met en relief son utilité (que Bloch formule sous forme d'euphémisme : «je ne puis me persuader que celui-ci doive être tout à fait inutile ») pour ses allocutaires idéaux, les chercheurs qui, à l'avenir, se pencheront sur l'histoire de ce qui n'est pas encore appelé la Seconde Guerre mondiale.

11 L'auteur veut en effet apporter sa contribution à une meilleure compréhension, dans le futur, des événements qu'il vit au présent. Son apport est d'autant plus précieux que les autorités, aussi bien françaises que allemandes, font un considérable travail de propagande pour s'assurer la sympathie - ou tout du moins, la docilité - de la population, et les matériaux qui en sont issus pourraient fort bien servir à réécrire l'histoire telle qu'elle s'est réellement passée. Troisième caractéristique du témoignage de Marc Bloch: ce dernier sert donc de contre-discours, en ce qu'il se définit par " proximité et différenciation par rapport à d'autres discours » (Doury, Quet et Tseronis 2015 : §4), en l'occurrence les discours officiels expliquant les causes et les raisons de la défaite de 1940. 

métaphorique sous la forme de «brumes, qu'autour du plus atroce effondrement de notre histoire commencent, dès maintenant, à accumuler tantôt l'ignorance et tantôt la mauvaise foi ». Bloch a d'ailleurs une conscience aigüe que son contre-discours peut éveiller la suspicion des autorités, voire même l'antagonisme d'une partie de la population. En écrivant son "témoignage", l'écrivain se range aux côtés de ces Français qui refusent de "cesser le combat ", comme l'exigeait le Maréchal Pétain dans son discours du 17 juin 1940, cinq jours avant de signer l'armistice. Cela suffit à le désigner comme ennemi aux yeux des nazis, ainsi que du gouvernement français de Vichy. façon certaine le destin de ce contre-discours, qu'il imagine néanmoins occulte: de manuscrit circulant «sous le manteau ", il pourrait devenir l'un des «dossiers cachés [qui] s'ouvriront » le jour où « la liberté de pensée et de jugement » sera de nouveau de mise. La publication immédiate est, quant à elle, inenvisageable. En effet, Bloch n'a pas recours à cet art « d'écrire entre les lignes » (Strauss 2003 : 25) qui caractérise certains textes de Maïmonide ou Spinoza et qui aurait pu lui permettre de publier de son vivant, au nez et à la barbe des autorités. Au contraire, son témoignage est direct et franc (une «franchise » qui pourrait d'ailleurs passer pour "un peu rude»), et ambitionne de n'être pas moins que le «procès-verbal de l'an 1940 ", c'est-à-dire une relation exacte et exhaustive des faits qui se sont déroulés au cours de cette année charnière.

14 de se présenter pour déposer : «Avant même de faire le point de ce que j'ai pu voir, il convient de dire avec quels yeux je l'ai vu ». Le témoignage repose en effet sur une double condition, à l'origine d'un paradoxe apparent. D'une part, on demande au témoin d'intervenir en tant qu'individu, que «je » incomparable, pour garantir avoir bien été présent - lui, et non un autre - au moment des événements qu'il s'apprête à relater. En même temps, le témoin est sommé de faire preuve de la plus extrême objectivité, c'est-à-dire qu'il doit s'engager à relater les faits sans les déformer ou les filtrer au tamis de ses intérêts, de ses croyances ou de sa sensibilité.

C'est donc une subjectivité maîtrisée qui est exigée du témoin, et Bloch en a conscience. Du point de vue énonciatif, le locuteur veille ainsi à prendre en charge sa propre parole, comme l'indique l'usage omniprésent du pronom personnel «je ». Ce «je » est incarné (" avec quels yeux je l'ai vu») et rattaché à une personne dont le corps et l'esprit ont été pleinement mobilisés au moment où les événements ont été vécus, au point d'en être affectés (« combien il me semblerait plus commode de céder aux conseils de la fatigue et du découragement!»). Néanmoins, parce que le témoignage implique que cette subjectivité soit contenue et tende, de façon que l'on pourrait qualifier d'asymptotique, vers une forme d'objectivité, Bloch prend soin de cantonner la place prise par ce « je ». Sans qu'il soit totalement minoré comme dans l'écriture "blanche » et dépersonnalisée des témoignages de certains rescapés des camps de concentration (Amossy 2004 : \$11), le « je » est à plusieurs reprises agrégé au destin collectif de tous les autres témoins potentiels qui ont partagé la même expérience. Marc Bloch est ainsi un soldat "parmi beaucoup" et dont le "destin [est] commun [...] avec celui de presque toute [sa] génération ». 


\section{La légitimation du témoignage à travers la construction d'un ethos testimonial}

l'argumentation pour légitimer son témoignage. Et pour cause : il sait que son discours sera examiné à l'aune de ce qu'il appelle son "état-civil», et ce que l'analyse du discours identifie comme ethos préalable ou prédiscursif (Maingueneau 2002 : 58). Cette notion recouvre « l'ensemble des données dont on dispose sur le locuteur au moment de sa présentation de soi » (Amossy 2010 : 73). Les données en question sont de nature très diverse, comme la représentation sociale ou la représentation individuelle, mais ont pour dénominateur commun de pouvoir influer sur la perception que les allocutaires auront du discours. En l'occurrence, Bloch a une connaissance fine de l' ethos préalable qui lui est attaché, ainsi que des réactions qu'il pourra provoquer chez ses lecteurs. C'est pourquoi il va s'attacher à présenter plusieurs facettes ou figures de sa personnalité et à en désamorcer les aspects problématiques en leur surimprimant un ethos discursif de témoin qualifié.

\section{La figure de l'historien}

19 Marc Bloch commence par présenter son "métier », qui est d' "écrire et enseigner l'histoire». Pratiquée de nombreuses années, cette activité lui a permis d'affiner certaines qualités ou « habitudes » qu'il entend mettre, de façon explicite, au crédit du témoin qu'il est. Quelles sont ces habitudes? Parce que l'historien est amené à "feuilleter beaucoup de documents d'âges divers", il est rôdé à la "critique », à "l'honnêteté », et à faire "le tri du vrai et du faux ». Il s'agit là d'un premier pas essentiel dans la construction d'un ethos de témoin, dans la mesure où ces compétences

Argumentation et Analyse du Discours, 21 | 2018 
traduisent toutes une forme de recherche ou d'appétence pour la vérité. Dire le vrai est en effet le but ultime fixé au témoin, tel que Bloch le définit dans son discours de 1914 et tel que le conçoit la procédure judiciaire traditionnelle à laquelle l'historien se réfère constamment. Il suffit ainsi de rappeler le contenu du serment qui, en France, est alors exigé de celui qui monte à la barre pour déposer : «Je jure de parler sans haine et sans crainte, de dire toute la vérité, rien que la vérité ».

Le second ensemble de qualités a, quant à lui, trait à l'observation. Un historien doit "beaucoup regarder ", aussi bien les « vieux grimoires » du passé que la nature et les événements du présent. Bloch estime ainsi que l'historien des campagnes doit avoir « de bons yeux » afin de contempler la forme des champs. Cette dernière précision est loin d'être anodine. En effet, l'intellectuel, et à plus forte raison l'historien, est souvent pris pour cible de critiques sur son incapacité (ou sa répugnance) à sortir de sa tour d'ivoire pour se confronter au monde réel. Il a conscience de ces préjugés, et souhaite montrer que ses dons d'observation ne sont pas mis au service d'une analyse abstraite et stérile, mais d'une meilleure compréhension de « la vie ».

Qui plus est, un bon historien doit être capable d'évoluer avec agilité sur l'échelle reliant le passé au présent. L'auteur enrichit ainsi le lieu selon lequel le passé éclaire le présent d'un enseignement inversé : « sans se pencher sur le présent, il est impossible de comprendre le passé ». Loin d'être un chercheur en robe de chambre, le témoin Marc Bloch va dans les champs, les connait, les estime, a le coup d'œil propre à en percer les secrets, à l'image du paysan héritier d'une science plurimillénaire. Le souci heuristique et méthodologique de l'historien se transforme ainsi en acquis ontologique pour le témoin.

\section{La figure du soldat}

Bloch continue la construction de son ethos discursif de témoin valable en décrivant son expérience de soldat. En effet, L'étrange défaite se veut un témoignage sur les causes de la débâcle militaire française, ce qui nécessite une expérience réelle des «différents aspects de la nation en arme ». L'auteur prend soin de préciser que son destin est ici commun avec celui de toute sa génération, laissant deviner une multitude de témoins potentiels prêts à corroborer sa version des faits.

23 Là où, en revanche, son expérience se distingue, c'est dans son étendue, qualifiée « d'assez exceptionnelle ». En effet, Bloch a participé à deux guerres, et a occupé un nombre important de fonctions aux différents échelons de la hiérarchie militaire, de " simple soldat » à membre d'un "état-major d'armée ». Cette précision biographique permet d'ajouter à l'ethos du témoin, outre les qualités d'analyse, d'honnêteté et d'observation, celle de l'expérience, venue avec la grande "variété » de situations auxquelles il a été confronté. Le témoin Marc Bloch ne sait donc pas seulement parler ; il sait aussi de quoi il parle.

\section{La figure du brave}

En plus d'avoir participé à deux guerres, il s'y est également distingué. C'est ce qui ressort de la description donnée à l'avant-dernier paragraphe de notre corpus, et qui débute par la relation d'un échange que l'auteur a eu avec un jeune officier. Ce dernier lui fait la confidence, sur le pas d'une porte et alors que la ville est en train d'être bombardée, qu'il perçoit l'historien comme « un guerrier », bien qu'il soit un « civil ». 
Si Bloch estime que la formule "peut paraitre naïve ", il ne la croit néanmoins pas "tout à fait fausse». Ce sentiment est corroboré par une seconde anecdote, dans laquelle un médecin militaire fait remarquer au "vieux professeur " qu'il possède plus que personne «l'esprit militaire». Ces deux témoignages sur le témoin sont d'autant plus imposants et moins sujets à caution qu'ils viennent d'un officier et d'un docteur de l'armée, c'est-à-dire d'individus qui sont a priori légitimés à jauger la valeur martiale d'un soldat.

Ce jugement de bravoure est en outre confirmé par l'obtention de quatre citations lors de la guerre de 14-18, et la certitude du témoin qu'il aurait pu obtenir « un ruban de plus sur [sa] vareuse» si les Allemands n'avaient pas mis rapidement fin aux opérations. Enfin, le caractère combatif de Marc Bloch est de nouveau souligné lorsqu'il rappelle qu'il n'a jamais rechigné à retourner à la guerre, déjà en 1915 alors qu'il sortait de convalescence, puis en s'étant « laissé maintenir en activité » alors que son âge et ses six enfants lui auraient permis de pendre son uniforme à un clou.

En plus de la capacité d'analyse et de la variété de l'expérience, c'est donc la bravoure martiale qui est ici revendiquée. Elle doit conduire le lecteur à deviner chez Marc Bloch un autre type de valeur: le "courage de la vérité" (Foucault 2009), une qualité essentielle pour l'ethos du témoin, notamment lorsque celui-ci produit un contrediscours opposé à la propagande officielle. Au-delà, si l'historien insiste autant sur sa contribution active et volontariste à l'effort militaire français, c'est qu'il sait qu'en tant que Juif, il devra répondre à un tout autre type d'accusation : celui de "parti pris » anti-français.

\section{Le contre-discours patriotique comme source de légitimation}

\section{Le Juif : un Français privé de légitimité}

Observateur, expérimenté et courageux : en dressant son propre portrait, Marc Bloch a glané une série de caractéristiques permettant de façonner son ethos de témoin légitime. Mais la description ne s'arrête pas là, et l'historien y ajoute une quatrième figure : son identité juive. Celle-ci n'a pourtant, de prime abord, aucun rapport avec le statut de témoin et les qualités que l'on pourrait exiger de lui. Mais c'est sans compter sur le fait que, comme le redoute l'auteur, " peut-être les personnes qui s'opposeront à [son] témoignage chercheront [...] à le ruiner en [le] traitant de " métèque " ".

Le choix du terme "métèque » comme anticipation de la critique est fondamental dans la compréhension de l'argumentation développée par Bloch. Issu du grec "métoïko » (celui qui a changé de résidence), son emploi se développe à la fin du $19^{\mathrm{e}}$ siècle et est propagé par des auteurs nationalistes comme Charles Maurras, avec une connotation fortement xénophobe et injurieuse. Il désigne alors l'immigré ou le descendant d'immigrés installé en France, auquel on refuse de façon symbolique le droit de se sentir français en le rappelant à son origine étrangère. Son usage est également répandu à l'armée, à l'époque où Bloch vit et écrit. Léon Aréga, ancien combattant du $22^{\mathrm{e}}$ régiment de la légion étrangère, utilise ainsi ce terme particulièrement chargé pour apostropher les Français au nom de ses camarades massacrés: "La guerre vous a délivrés des maroquiniers "polonais" qui vivaient de rien et travaillaient pour rien. Dès 
ce jour, la bonne maroquinerie parisienne va retrouver la splendeur de jadis, avant l'invasion des métèques » $(1946: 77)$.

Ce que révèle l'emploi du terme "métèque » c'est que le judaïsme est, aux yeux des contempteurs que Bloch anticipe, fondamentalement incompatible avec la qualité de Français. Et c'est parce qu'un Juif est un Français illégitime que sa parole, lorsqu'elle porte sur des sujets intéressants la communauté nationale dans ce qu'elle a de plus sensible, comme l'Armée, et de plus honteux, comme la défaite, doit être discréditée d'office. Il faut ici faire intervenir des éléments de contexte. L'affaire Dreyfus a éclaté un demi-siècle auparavant, mais elle continue de marquer les esprits, et les mentalités qui lui ont donné naissance ne sont pas éteintes. Les Juifs de France, qu'ils soient nationaux, immigrés ou apatrides, continuent d'être perçus comme des traîtres en puissance, quand bien même ils ont payé le prix du sacrifice au cours de la Première Guerre mondiale.

Ces préjugés permettent d'ailleurs d'expliquer une bizarrerie dans l'agencement de l'argumentation. Avec l'origine juive, ce sont quatre facettes de son ethos préalable que Bloch va détailler pour mieux élaborer son ethos discursif de témoin. Le lecteur s'attendrait à voir la description du brave suivre celle du soldat, le premier étant un qualificatif fréquemment rattaché au second. L'auteur intercale néanmoins le Juif entre les deux. Une première explication voudrait qu'en finissant par la qualité du brave, Marc Bloch s'autorise à clore son argumentation sur un élément moins polémique que le judaïsme, afin de préserver au maximum l'adhésion de ses allocutaires pour la suite du témoignage. Une autre lecture, complémentaire, est permise par le lien découvert entre identité juive et appartenance nationale, aux yeux des contempteurs de Marc Bloch. Si la figure du militaire courageux et prêt au sacrifice patriotique suit celle du Juif, c'est pour mieux contrecarrer le cliché antisémite selon lequel les Juifs seraient des lâches et des traîtres en puissance.

\section{L'élaboration d'un contre-discours de patriote}

31 Comme nous l'avons vu, pour déposer, un témoin a besoin de légitimité, et Marc Bloch tâche de l'acquérir à travers l'élaboration d'un ethos discursif conforme. Ce que révèle l'évocation de l'identité juive de l'auteur, c'est que cet ethos n'est pas seulement construit en prenant pour modèle une image idéalisée et normative de ce que devrait être un témoin. Il en incorpore certes plusieurs éléments, comme la nécessité de connaître directement les faits (ce qui relève du soldat), de savoir les analyser (c'est la spécialité de l'historien) ou d'oser les dire (ce que l'on peut exiger du brave). Mais de façon fort intéressante, cet ethos discursif est également taillé en anticipant l'image qu'un détracteur potentiel pourrait se faire d'un témoin valable dans la situation présente.

Plutôt que de l'ignorer purement et simplement, ou de mentionner qu'elle n'a aucun rapport avec le sujet et les enjeux traités, Bloch anticipe ainsi l'attaque ad hominem et la prend en compte dans la construction de son ethos. C'est ainsi qu'il entreprend d'élaborer un contre-discours patriotique visant à légitimer sa capacité à dire cet autre contre-discours que constitue son témoignage. Cette argumentation se fait en deux temps : la minimisation de son identité juive, et l'accentuation de son ethos de patriote français. 


\section{Une identité juive revendiquée, mais minimisée} juif » ouvre le paragraphe, à la manière du témoin qui décline ses noms et qualités. Décliner peut ici être compris dans ses deux acceptions : celle de présenter une identité face à une personne qui la demande, mais aussi celle de la repousser. En effet, immédiatement après avoir fait l'aveu de son judaïsme, l'auteur introduit une concession : il est Juif «sinon par la religion, qu['il] ne pratique point non plus que nulle autre, du moins par la naissance ». Son identité juive est minimisée, reléguée à une simple « origine ». Il précise ainsi qu'il n'est pas un Juif par la croyance, évacuant la possibilité que ses convictions de Français puissent être influencées ou entachées.

Il prend ensuite soin de noter qu'il ne tire « ni orgueil ni honte » de ce qui est présenté comme un simple état de fait. Cette absence d'affects revendiquée permet d'anticiper le double reproche qu'un opposant xénophobe pourrait être tenté de lui faire : fier d'être Juif, il serait un Français imparfait, aux allégeances multiples, et que l'on devrait soupçonner; honteux d'être Juif, il faillirait à son honneur, pourrait avoir quelque chose à cacher, et serait donc un Français louche et de peu de valeur. L'auteur veille ainsi, tout au long de ces lignes où il décrit son rapport au judaïsme, à garder un ton neutre, descriptif et presque scientifique, ce qui lui est d'autant plus aisé qu'il peut exhiber sa qualité de «bon historien ».

Quand l'homme Marc Bloch rejette les croyances juives, le scientifique s'attaque lui aux "prédispositions raciales", qu'il qualifie de "mythe » pour mieux les balayer, et à la notion de "race pure ", une "absurdité particulièrement flagrante». Les Juifs des premiers temps sont en effet décrits, de façon légèrement péjorative, comme un simple "groupe de croyants, recrutés, jadis, dans tout le monde méditerranéen, turco-khazar et slave ». Si les Juifs ont été "recrutés », c'est qu'ils étaient auparavant des individus " comme les autres ». De plus, les faire venir de régions aussi variées empêche de les rattacher à une terre spécifique (comme la tradition juive le revendique par ailleurs, avec la notion de "Terre promise »). Parce qu'ils sont de partout, les Juifs sont en fait de nulle part, et cette absence d'attaches devrait leur permettre de s'enraciner plus facilement et plus profondément dans le terreau français. Il faut noter ici qu'à cette date, le terme de race semble recouvrir celui de peuple, puisqu'on retrouve cet usage interchangeable chez plusieurs auteurs de la fin $\mathrm{du} 19^{\mathrm{e}}$ siècle et de la première moitié du $20^{\mathrm{e}}$ siècle, à l'image d'Ernest Renan qui utilise les deux mots de façon indistincte dans sa conférence consacrée au « judaïsme comme race et comme religion » $(1883: 2)$.

Bloch ne se reconnaît donc Juif ni par la religion (à laquelle il n'adhère pas), ni par les affects (auxquels il est indifférent), ni par la race/le peuple (qui n'existe pas). Il ne l'est que par la naissance, et s'il n'y a pas ici de reniement, la revendication de son identité est strictement reléguée à ce qu'impose l'honneur: «je ne revendique jamais mon origine que dans un cas : en face d'un antisémite ».

\section{Un ethos de Français accentué}

Cette conception minimale et défensive de l'identité juive tranche avec la pompe de l'argumentation patriotique qui suit. Pour répondre à l'insulte (anticipée) de "métèque », Bloch va élaborer un ethos discursif de Français dont les ressorts argumentatifs sont particulièrement riches. L'auteur commence par la formule « je leur répondrai, sans plus ", parce que les arguments qui vont suivre sont tellement évidents 
qu'ils ne devraient pas avoir à être rappelés. Cette entrée en matière feutrée tranche d'ailleurs avec le caractère hyperbolique des arguments qu'il utilise. Autre fait remarquable: les éléments mobilisés pour légitimer son ethos de Français sont rigoureusement les mêmes que ceux utilisés pour minimiser son identité juive, mais inversés. Ce jeu spéculaire fait ainsi intervenir six composantes :

- La filiation: là où l'inscription dans une généalogie juive n'est que furtivement évoquée, les exploits patriotiques des aïeux sont eux rappelés dans le détail, de l'arrière-grand-père soldat pour la France en 1793, au père qui servit en 1870 dans Strasbourg assiégé. En outre, la filiation ne prête pas à conséquence dans le cas du judaïsme ; en revanche, la gloire familiale passée est rappelée pour mieux rejaillir sur l'auteur dans le cas de la revendication identitaire française.

39 - Le ton: si l'auteur est sec et parfois cassant lorsqu'il parle du judaïsme, il se fait infiniment plus lyrique lorsqu'il s'agit de décrire l'amour porté à la patrie. Il multiplie ainsi les métaphores champêtres («j'ai bu aux sources de sa culture», «je ne respire bien que sous son ciel », « la patrie dont je ne saurais déraciner mon cœur »), que l'on s'attendrait davantage à trouver au cœur d'un sonnet que dans un témoignage ou un travail d'historien.

- Les affects : alors que son rapport au judaïsme est dénué d'émotions, aussi bien négatives que positives, celui qui le lie à la France en est saturé, comme en témoignent le rappel des «fervents mainteneurs » des traditions nationales, auxquels il appartient, et l'angoisse que l'on devine à l'idée d'une expulsion du pays.

41 - Le culte : quand la religion a déserté l'identité juive de Bloch, elle prospère dans son ethos de Français, notamment lorsqu'il rappelle avoir «été élevé dans le culte [des] traditions patriotiques ».

- La terre : si le Juif est issu d'un "groupe de croyants » sans attaches, le Français a quant à lui un rapport fusionnel à la terre. La patrie n'est pas un concept théorique, mais une réalité sensible et charnelle, et le témoin a bien soin, tout au long du corpus, de rappeler son amour et sa profonde connaissance de la terre nourricière française. Cette revendication n'est d'ailleurs pas exclusive à Marc Bloch ; on se rappellera que le célèbre discours du 25 juin 1940, dans lequel le Maréchal Pétain affirmait que « la terre, elle, ne ment pas» a été rédigé par l'écrivain juif Emmanuel Berl. Enfin, cet attachement à la terre s'accompagne de la crainte de devoir en être exilé, et de la promesse d'y rester toujours attaché - reprenant ainsi un thème classique dans la tradition et la liturgie juives.

43 - Le vocabulaire : quand Bloch traite de son identité confessionnelle, il emploie le terme «juif ». En revanche, dès qu'il passe sur le terrain patriotique, il adopte le terme euphémistique "d'Israélite ", qui est typiquement français. Le choix de ce vocable n'est pas anodin. En recourant à la dénomination utilisée par le groupe de référence pour se définir lui-même, le locuteur montre son appartenance à ce même groupe, ce que souhaite réaliser l'auteur avec les patriotes français.

Pour résumer, il accorde à son identité française tout ce qu'il retire à son identité juive. C'est donc par un effet de contraste argumentatif que l'historien va construire son ethos de Français ou de «bon Français », pour reprendre une formule qu'il utilise dans son Testament spirituel, écrit une année plus tard (1945). 


\section{Conclusion}

L'étrange défaite, devenu un classique pour la compréhension du métier d'historien et pour l'histoire de la Seconde Guerre mondiale, n'ont pas manqué de s'interroger sur la portée et l'usage scientifiques que l'on pouvait faire de ce document. Dominique Damamme y voit ainsi un exemple «d'expertise auto-instituée » (2000: 112), c'est-àdire une combinaison de pratiques politiques et de pratiques scientifiques. Jacques le Goff (1993 : 12) estime, quant à lui, que le fondateur des Annales a fait ici «vraiment œuvre d'historien, non de journaliste ». Pour autant, la question du genre discursif auquel cet ouvrage appartient, indépendamment de la revendication de son auteur, semble avoir été délaissée. Certains chercheurs ont ainsi pu développer tout un cadre énonciatif du témoignage basé sur la " consigne de traitement " (Wisard 1994 : 12) que donne Bloch de son propre texte, sans s'interroger sur son adéquation aux conditions qui régissent ce genre de discours.

travail ne pourra pas être réalisé ici, mais nous pouvons d'ores et déjà remarquer que "pour l'argumentation "ordinaire" comme pour l'argumentation juridique, la bonne foi du témoin, le fait qu'il n'ait aucun intérêt matériel, affectif ou idéologique à affirmer ce qu'il dit, sont des conditions premières d'acceptabilité du témoignage " (Doury 1999 : 169). Notre étude a permis de mettre en évidence que, pour répondre aux attaques visant à ruiner son témoignage, Marc Bloch déploie un contre-discours patriotique, au sein duquel il élabore un ethos discursif de «bon Français ». Ainsi, pour mieux se conformer à l'ethos du témoin tel qu'il s'imagine que ses contradicteurs se la figurent, il va aller jusqu'à légitimer l'objectivité de son témoignage par la subjectivité de son sentiment patriotique. Plutôt que de le disqualifier, son "intérêt affectif et idéologique " est ainsi censé devoir soutenir son exercice. L'étrange défaite est à cet égard un bien étrange témoignage.

Second enseignement: le texte de Bloch est révélateur de l'influence des idées antisémites de l'époque, à un degré particulièrement prononcé. C'est notamment le cas du soupçon de double allégeance, qui influence, de manière implicite, l'ensemble de l'argumentation. L'historien écrit avec, en tête, l'idée que des antisémites se trouveront parmi ses allocutaires. En témoigne le très simple usage de la formule «je suis juif », quand on sait que Bloch «ne revendique jamais [son] origine juive que dans un cas : en face d'un antisémite ». Cette anticipation de l'antisémitisme chez certains au moins de ses lecteurs futurs est d'autant plus frappante que l'auteur se montre, par ailleurs, optimiste quant à l'issue de la guerre et au changement des mentalités qui s'ensuivra. Il écrit ainsi, dès les premières pages de son ouvrage : «Un jour viendra, tôt ou tard, j'en ai la ferme espérance, où la France verra de nouveau s'épanouir, sur son vieux sol béni déjà de tant de moissons, la liberté de pensée et de jugement ». Si l'auteur conçoit que la censure et le totalitarisme soient appelés à disparaître, ce n'est pas le cas de l'antisémitisme. Se voulant un témoignage de Marc Bloch, ce passage constitue aussi un témoignage sur la force des discours antijuifs de l'époque, et leur imprégnation dans les esprits. 


\section{BIBLIOGRAPHIE}

Amossy, Ruth. 2004. «L'espèce humaine de Robert Antelme ou les modalités argumentatives du discours testimonial », Semen 17, 131-148

Amossy, Ruth. 2010. La présentation de soi. Ethos et identité verbale (Paris : PUF)

Amossy, Ruth. 2016 [2012]. L'argumentation dans le discours (Paris : Colin)

Aréga, Léon. 1946. Comme si c'était fini (Paris : Gallimard)

Bloch, Marc. 1945. « Testament spirituel », rédigé le 18 mars 1941, Annales. Histoire, sciences sociales $8: 1,6-9$

Bloch, Marc. 1950. «Critique historique et critique du témoignage », discours de distribution des prix prononcé au lycée d'Amiens le 13 juillet 1914, Annales. Economies, sociétés et civilisations $5: 1$, $1-8$

Bloch, Marc. 1990 [1946]. L'étrange défaite (Paris : Gallimard)

Damamme, Dominique. 2000. «Un cas d'expertise, l'étrange défaite de Marc Bloch », Sociétés contemporaines 39 , 95-116

Danblon, Emmanuelle. 2010. « La rhétorique : art de la preuve ou art de la persuasion? », Revue de métaphysique et de morale 66, 213-231

Doury, Marianne. 1999. « Les procédés de crédibilisation des témoignages comme indices des normes argumentatives des locuteurs ", Rigotti, Eddo (éd.). Rhetoric and Argumentation, Proceedings of the International Conference Lugano 1999 (Tübingen : Niemeyer), 167-180

Doury, Marianne. 2009. «Acte et personne dans l'argumentation : le cas du procès d'intention », Ribeiro, Henrique Jales (éd.). Rhetoric and Argumentation in the Beginning of the XXIst Century (Coimbra : Imprensa da Universidade de Coimbra), 165-179

Doury, Marianne, Mathieu Quet \& Assimakis Tseronis. 2015. « Le façonnage de la critique par les dispositifs. Le cas du débat sur les nanotechnologies », Semen 39, 39-58

Dulong, Renaud. 1998. Le témoin oculaire. Les conditions de l'attestation personnelle (Paris : Editions de l'EHESS)

Fleury, Béatrice \& Jacques Walter. 2012. « Carrière testimoniale : un opérateur de la dynamique mémorielle et communicationnelle », ESSACHESS. Journal for Communication Studies, $5: 2$, 153-163 Foucault, Michel. 2009. Le Courage de la vérité (Paris : Seuil)

Le Goff, Jacques. 1993. «Préface » à Apologie pour l'histoire ou Métier d'historien de Marc Bloch (Paris : Colin)

Maingueneau, Dominique. 2002. « Problèmes d'ethos », Pratiques 113-114, 55-67

Mausen, Yves \& Thomas Gomart. 2000. « Témoins et témoignages », Hypothèses 3, 69-79

Renan, Ernest. 1883. Le Judaïsme comme race et comme religion. Conférence faite au Cercle Saint-Simon le 27 janvier 1883 (Paris : Calmann Lévy)

Strauss, Leo. 2003 [1952]. La persécution et l'art d'écrire, trad. Olivier Sedeyn (Paris : Editions de l'Eclat) 
Wisard, François. 1994. «L'énonciation d'un témoignage. L'étrange défaite de Marc Bloch », Bulletin de l'Institut de Linguistique et des Sciences du Langage de la Faculté des Lettres de l'Université de Lausanne $14,7-140$

\section{RÉSUMÉS}

Cet article étudie les modes discursifs par lesquels l'historien Marc Bloch élabore un ethos testimonial dans l'introduction de son Etrange défaite. L'ouvrage retrace l'expérience de l'auteur de la défaite de l'armée française en 1940 et en analyse de façon très fouillée les causes, se posant ainsi en contre-discours face à la propagande officielle en vigueur. Parce qu'un témoin doit légitimer sa prise de parole, Marc Bloch entreprend de décliner son état-civil de façon à satisfaire aux exigences du modèle testimonial traditionnel issu de la rhétorique et du droit. Au cours de son argumentation, il revient sur son identité juive, une composante qui n'a, a priori, aucun lien avec le sujet de son ouvrage. Ce faisant, il nous oblige à reconsidérer d'un œil neuf les mécanismes de revendication, de construction et de limitation d'un ethos discursif de témoin face à des stéréotypes.

This study aims at analyzing the discursive means by which the French historian Marc Bloch builds an ethos of witness in the introduction of Strange Defeat. This book recounts the author's experience of the defeat of the French army in 1940; by thoroughly examining its causes, it offers a counter-discourse to the official propaganda of the time. To legitimize his status as a witness, Bloch exposes elements of his biography in a manner that is consistent with the traditional rhetorical and legal definitions of the position. Over the course of his argumentation, he explicitly references his Jewish identity, an element that has no a priori connection with the focus of his book. Thus, he forces readers to reconsider the mechanisms responsible for both constructing, and limiting, the ethos of a witness, when confronted with prejudices.

\section{INDEX}

Mots-clés : contre-discours, ethos, judaïsme, légitimation, témoignage

Keywords : counter-discourse, ethos, judaism, legitimization, testimony

\section{AUTEUR}

\section{JONATHAN SITBON}

Université de Tel-Aviv, ADARR 